\title{
Prospettive di fine vita dei parchi eolici (wind farms) nelle politiche dell'economia circolare
}

\author{
Esteban Poviña ${ }^{1}$ \\ ${ }^{1}$ Affiliation not available
}

\begin{abstract}
L'energia eolica negli ultimi anni ha avuto un forte incremento e la dismissione degli impianti eolici già esistenti sta suscitando molto interesse sul possibile recupero delle risorse. Una turbina eolica ha una durata media di vita tra 20-25 anni, e oggi 34,000 turbine in Europa hanno 15 anni o più. Una stima di 14,000 pale potrebbe essere disattivata entro il 2023, un equivalente tra 40,000 e 60,000 tonnellate. Pertanto, un mercato potenziale per le turbine eoliche fuori uso e i loro prodotti recuperati deve essere stabilito in Europa.

Circa l'85-90\% della massa totale delle turbine eoliche può essere riciclata. Il problema principale rimane sulle pale, che sono realizzate in materiale polimerico rinforzato con fibre, i cui metodi di riciclaggio non sono tecnologicamente sviluppati.
\end{abstract}

La situazione di fine vita di un parco eolico richiede una decisione tra differenti possibili progetti sostenibili per l'medio ambiente. La decisione finale sarà influenzata da diversi fattori.

\section{Contesto energetico}

A causa dello sviluppo delle economie mondiali, la domanda di energia è incrementata ogni anno più velocemente. L'industrializzazione dei paesi e l'aumento della popolazione mondiale hanno prodotto un'intensificazione dell'utilizzo di combustibili fossili per la produzione di energia ${ }^{1}$. 
L'uso dei combustibili fossili per la produzione di energia ha diversi impatti ambientali negativi, principalmente a causa dell'elevata quantità di emissioni di gas a effetto serra. Diversi studi dimostrano che queste emissioni inquinanti sono responsabili del riscaldamento globale, dell'innalzamento del livello del mare, dell'inquinamento atmosferico, dell'emissioni odorifere indesiderate, delle precipitazioni acide e dell'emissione di sostanze radioattive ${ }^{2,3,4,5,6,7}$.

Alla luce di questa problematica, il modo di agire per salvaguardare il nostro pianeta sembrerebbe quello legato all'utilizzo delle fonti rinnovabili, che sono considerate inesauribili e compatibili con l'ambiente ${ }^{8}$. L'UE mira ad essere neutrale dal punto di vista climatico entro il 2050 con emissioni nette di gas a effetto serra pari a zero. Questo obiettivo sarà raggiunto seguendo le politiche stabilite dall'accordo "European Green Deal”. Secondo l'International Renewable Energy Agency (IRENA), per raggiungere tali obiettivi, le energie rinnovabili dovrebbero fornire due terzi dell' approvvigionamento energetico globale entro il 2050, principalmente attraverso il fotovoltaico e l'energia eolica.

Inoltre, si deve considerare che le fonti rinnovabili hanno anche forti impatti nel medio ambiente, e le valutazioni su quest'ultimi devono essere svolte prima di stabilire un progetto ${ }^{1}$. In particolare, la selezione dei progetti costituisce un problema decisionale complesso, che può essere affrontato con l'analisi decisionale multicriteri (MCDA) ${ }^{9}$. Ulteriormente, una procedura di valutazione dinamica mira ad aiutare i pianificatori quando si tratta di prendere in considerazione potenziali danni all'ambiente dovuti a progetti energetici. Questa valutazione ambientale è in grado di garantire informazioni più efficaci sui contenuti del progetto, con l'obiettivo di portare le scelte verso azioni che possano raggiungere la piena compatibilità e sostenibilità ambientale, con il più alto consenso della comunità ${ }^{10}$.

Al fine di stabilire normative ecologiche come il Green Deal, sono indispensabili la combinazione dei principi fondamentali del nesso tra la produzione di energia ed il consumo associato di acqua, e degli approcci tecnologici emergenti per individuare e proporre soluzioni nel campo della 
protezione gestione delle risorse per l'ambiente ${ }^{11,12,13,14}$.

Ponendo particolare attenzione sull'energia eolica in questo cambiamento radicale del mercato energetico, bisogna effettuare valutazioni su tutto il ciclo di vita di un parco eolico. È necessario analizzare i potenziali impatti sulla vegetazione, flora e fauna, consumo di acqua, ecc. che possono verificarsi nelle tre fasi di vita del progetto: costruzione, operazione e dismissione ${ }^{15}$. La valutazione degli impatti è vitale per raggiungere gli obiettivi di sviluppo sostenibile intesi come migliori condizioni della qualità di vita senza modificare negativamente gli ecosistemi già esistenti. Pertanto, la valutazione d'incidenza ambientale deve contemplare diversi aspetti, dalla conservazione degli habitat fino alla gestione sostenibile dei rifiuti ${ }^{16}$.

Per illustrare questo passaggio alle fonti rinnovabili, l'energia eolica installata a livello mondiale è stata incrementata fortemente e i maggiori produttori sono l'Europa, gli Stati Uniti e la Cina . Secondo le statistiche di Wind Europe, L'Europa disponeva di 220 GW di potenza eolica installata entro la fine del 2020. Propriamente, nel 2020 sono stati installati 14.7 GW di nuova capacità eolica e si prevede di installare $105 \mathrm{GW}$ nei prossimi cinque anni.

\section{I parchi eolici}

Una turbina eolica, responsabile di trasformare l'energia cinetica del vento in energia elettrica, è composta da diversi elementi, e ogni componente è disegnato dettagliatamente con un certo materiale per svolgere un determinato ruolo. Secondo una valutazione del ciclo di vita di un parco eolico VESTAS di 100 MW, 25,000 componenti sono utilizzati in un modello VESTAS V112-3,45 MW.

Un parco eolico comprende anche i componenti convenzionali essenziali per il suo corretto funzionamento, tra cui il trasformatore dell'impianto, i cavi elettrici ed altri elementi elettromeccanici ${ }^{17}$. 
Secondo la Commissione Elettronica Internazionale, una pala eolica dovrebbe avere almeno una vita utile di 20 anni. Le turbine eoliche onshore hanno una durata media di 20-25 anni, mentre i nuovi modelli offshore possono arrivare fino a 30-35 anni di servizio.

In accordo a Wind Europe, oggi 34,000 turbine in Europa hanno 15 anni o più, che rappresentano $36 \mathrm{GW}$ di capacità eolica onshore. Si tratta di un volume significativo che necessita di una certa logistica per procedere con lo smantellamento, la raccolta, il trasporto, il trattamento dei rifiuti e il ristabilimento del sito in modo sostenibile.

Circa l'85-90\% della massa totale delle turbine eoliche può essere riciclata. La maggior parte dei componenti indicati utilizzano materiali comuni come calcestruzzo, acciaio e altri metalli, che hanno metodi di trattamento dei rifiuti ben definiti.

D'altra parte, le pale sono realizzate in materiale composito con una quantità tra $10-15$ tn/MW ${ }^{18}$. Di solito, sono composte per il $70 \%$ da una matrice polimerica termoindurente e per il $30 \%$ da E-Glass fibers. I metodi di riciclo di questi materiali non sono tecnologicamente sviluppati. Wind Europe stima che circa di 14.000 pale potrebbero essere smantellate entro il 2023, un equivalente a 40,000-60,000 tonnellate. Pertanto, le turbine eoliche fuori servizio rappresentano un'enorme quantità di materiale da trattare, e i diversi processi di trattamento devono essere definiti.

La situazione di fine vita richiede una decisione tra stabilire un progetto di repowering, tra l'estensione della vita del vecchio parco eolico e tra lo smantellamento del sito con un adeguato trattamento dei rifiuti.

\section{Repowering}

Repowering significa sostituire i vecchi modelli di turbine eoliche con quelli nuovi. Ci sono diversi vantaggi poiché la prima generazione di turbine eoliche può essere sostituita con moderne turbine eoliche multi-megawatt, e nei paesi che hanno iniziato presto con l'energia eolica, di solito le 
vecchie turbine eoliche sono collocate in luoghi in cui le condizioni di vento sono ottimali, e quindi le moderne turbine eoliche avranno prestazioni migliori, con un aumento della produzione energetica, minori impatti ambientali e un migliore sfruttamento delle risorse ${ }^{19,20}$.

I fattori economici e il complesso processo di autorizzazione sono i principali ostacoli. Gli elevati costi di investimento, dovuti alle opere civili di demolizione e costruzione delle infrastrutture, rappresentano un impedimento per i progetti di repowering ${ }^{21}$.

I regolamenti dei governi sono estremamente vitali per le decisioni di repowering. In alcuni paesi europei ci sono normative che ostacolano il repowering, contrariamente alle disposizioni della nuova direttiva sulle energie rinnovabili dell'Unione Europea; pertanto, gli incentivi economici sono necessari per lo sviluppo dei progetti di repowering.

\section{Estensione della vita utile}

L'estensione della vita si basa sulle condizioni operative durante la vita utile. È necessario una valutazione tecnica per determinare la vita utile rimanente della turbina e garantire che i livelli di sicurezza siano soddisfatti durante l'estensione della vita utile ${ }^{22}$.

La Danimarca dispone di una piattaforma di "Life Cycle Assestment" che rappresenta uno strumento di visualizzazione,tale da mostrare gli indicatori di prestazione ambientale per le turbine eoliche, attraverso un'analisi del ciclo di vita ${ }^{23}$. Questa piattaforma potrebbe essere anche utilizzata per stabilire un'operazione controllata delle turbine eoliche di ogni paese e definire le possibili opzioni di trattamento di rifiuti delle turbine eoliche.

Gli aspetti economici hanno un enorme impatto sulle decisioni di prolungamento della vita. In Germania, le sovvenzioni per l'energia eolica sono regolate dalle leggi sulle energie rinnovabili. I parchi eolici messi in funzione prima dell'aprile 2000, ricevono una tariffa speciale fino al 2020, indipendentemente dall'età delle turbine. Dopo il 2020, tutti i parchi eolici devono vendere la loro 
elettricità sul valore normale del mercato. Pertanto, prolungare la vita utile di un parco eolico per 5 anni potrebbe non essere redditizio, in quanto i capitali di investimento potrebbero non essere recuperati nei pochi anni di vita progettata ${ }^{22}$.

La decisione relativa all'estensione della vita utile è influenzata dallo stato di salute della turbina, dai requisiti per l'estensione della vita utile, dai regolamenti per il recupero dei crediti e dai regimi di sovvenzioni per i parchi eolici esistenti e nuovi. Se è economicamente redditizio e il sito è adatto per il repowering, il punto ottimale nel tempo per sostituire le vecchie turbine, deve essere determinato. Qualora il repowering non fosse possibile, l'estensione della vita dovrebbe essere valutata se i costi di capitale sono rimborsati alla fine della vita di progettazione ${ }^{22}$. Nel caso in cui entrambi non siano possibili da concretizzare, uno smantellamento sistemato con una logistica di classificazione dei rifiuti secondo il processo di trattamento da realizzare deve essere stabilito.

\section{Smantellamento delle turbine}

\section{Progetti di recupero}

Recupero significa re-utilizzare un componente in un'altra applicazione. Il calcestruzzo, i metalli ed altri materiali non hanno molte applicazioni di recupero in quanto non è economico né ambientale trasportare grandi quantità di materiale su lunghe distanze quando i processi di riciclo sono tecnologicamente sviluppati.

Nondimeno, le pale eoliche hanno buone potenzialità per essere utilizzate come componenti strutturali in diverse applicazioni. La Queen's University of Belfast ha sviluppato un progetto dove le pale eoliche sono state utilizzate come membri strutturali per un ponte pedonale ${ }^{24}$.

Da un'altra parte, Gentry et al., hanno svolto un progetto di "Housing" con le pale eoliche, concernente l'utilizzo dei pannelli per realizzare piattaforme di sollevamento, tetti, porte e finestre, ideale 
per case nei paesi in via di sviluppo in cui le condizioni di vita sono ancora precarie ${ }^{25}$.

Le pale anche possono essere utilizzati per fare mobili ${ }^{26}$, o segmentate in elementi discreti per applicazioni di calcestruzzo rinforzato per raggiungere un incremento delle proprietà meccaniche ${ }^{27}$.

\section{Riciclo dei materiali}

Il calcestruzzo può essere riciclato e riutilizzato come aggregato per nuove applicazioni strutturali ${ }^{28,29}$. L'acciaio è $100 \%$ riciclabile, ed anche gli altri metalli, attraverso tecnologie di riciclo sviluppati ${ }^{30,31}$. I materiali rari della terra possono essere estratti e riutilizzati per fare nuovi materiali magnetici, o fusi in una nuova combinazione ${ }^{32,33}$.

In riferimento ai materiali compositi, ci sono diversi metodi per il riciclo. Le diverse tecnologie di riciclo sono ancora sotto sviluppo ed alcune non possono competere con altre pratiche di trattamento, a causa sia dei prodotti ottenuti dai processi che non presentano ancora buone proprietà meccaniche, sia dall'elevato costo del processo.

Inoltre, bisogna tenere presente, che la quantità di materiale composito proveniente dalle turbine eoliche, rappresenta solo il 10\% del totale dei rifiuti compositi stimati entro il 2025, che le pale sono di diverse caratteristiche e che saranno fuori servizio tra diversi anni ;quindi sarà una fonte di materiale bassa, discontinua e non omogenea. Questo rappresenta un problema importante per stabilire un' industria di trattamento dei rifiuti.

Nella seguente lista vengono elencati i vantaggi (+) e gli svantaggi (-) dei processi di riciclo:

1. Cement co-processing: processo termico, TRL 9. (+) Efficiente, basso costo e di applicazione commerciale. (-) Massimo potenziale non sfruttato, forma fisica originale delle fibre persa e matrice incinerata ${ }^{34,35}$

2. Mechanical grinding: processo meccanico, TRL 9. (+) Efficiente, basse emissioni di $\mathrm{CO}_{2} \mathrm{e}$ basso costo. (-) Riduzione delle proprietà meccaniche delle fibre, emissioni di polvere e molti 
rifiuti generati ${ }^{35,36,37}$.

3. Pyrolysis: processo termico, TRL 9. (+) Recupero energetico, prodotti di idrocarburi generati e bassa riduzione delle proprietà meccaniche nelle fibre di carbonio. (-) Riduzione delle proprietà meccaniche delle fibre di vetro e residuo di resina $38,39,40,41,42$.

4. High Voltage fragmentation: processo elettro-meccanico, TRL 6. (+) Scalabile per maggiori quantità, fibre di buone qualità. (-) Bassa efficacia ed elevato costo ${ }^{43,44}$.

5. Solvolysis: processo chimico, TRL 5/6. (+) Fibre di carbonio pulite, lunghezza completa e buone proprietà meccaniche; Sub-prodotto di resina chimica resina chimica prodotta. (-) Riduzione delle propietà meccaniche nelle fibre di vetro, elevata quantità di solventi utilizzati e elevato costo $45,46,47,48,49,50,40$.

6. Fluidized bed: processo termico, TRL 5/6. (+) Trasferimento efficiente di calore, recupero di energia, tollerante con contaminanti e nessuna formazione di resina residuale. (-) $\mathrm{Ri}$ duzione delle proprietà meccaniche delle fibre di vetro dovuto all'attrito ed elevato costo $40,35,51,52,53,54$.

7. Micro-Wave pyrolysis: processo termico, TRL 4/5. (+) Trasferimento di calore efficiente e buone proprietà meccaniche delle fibre.(-) Elevato costo ed ancora solo ha applicazione di laboratorio $40,55,56,57$.

\section{Dismissione}

Incenerimento e Landfilling sono le ultime opzioni nella gerarchia dei trattamenti, in quanto i rifiuti delle turbine, non vengono sfruttati per produrre nuovi sub-prodotti e presentano molti impatti negativi. Tuttavia, dato il basso costo, sono pratiche ancora utilizzate in alcuni paesi, in cui i metodi di riciclo non hanno applicazione commerciale. Pertanto, è necessario stabilire una politica globale per vietare queste pratiche e promuovere, mediante incentivi economici, metodi di trattamento più amichevoli con il medio ambiente. 


\section{Sviluppo e disegni futuri}

Insieme a questo sviluppo energetico, anno dopo anno le turbine eoliche hanno incrementato le loro dimensioni. Un maggiore diametro del rotore, e quindi componenti più grandi (generatore, gearbox, torre), significa una maggiore produzione energetica. Questo porta a un maggiore consumo dei materiali, ma ci si aspetta che con i nuovi modelli più tecnologici si possa ridurre il impiego di alcuni materiali.

È necessario ridurre la massa delle turbine, così da poter ridurre i costi di trasporto, di costruzione e soprattutto avere una minore quantità di rifiuti dopo la vita utile. Esistono diversi approcci:

- Sostituire il cemento per Ground Granulated Blad Slag in un 70\% nella fondazione. Questo sub-prodotto proveniente dal processo di elaborazione del ferro ha buone proprietà meccaniche ed è più leggero.

- Utilizzare acciai di migliore qualità. Per esempio, con il utilizzo di un'acciaio S500 nella torre invece di un $\mathrm{S} 355$, un risparmio di peso del $30 \%$ può essere raggiunto. Anche se si utilizzano materiali di alta resistenza, un disegno strutturale della torre più leggero potrebbe essere implementato ${ }^{58}$.

- Disegni più leggeri della torre sono ancora sotto studio, utilizzando lamiere trapezoidali. I risultati ottenuti da un studio hanno dimostrato che con una torre trapezoidale è possibile ottenere una riduzione di peso fino al $20 \%$ rispetto ad una torre tubolare in acciaio di 90 $\mathrm{m}^{59}$.

- Generatori che utilizzano materiali superconduttori ad alta temperatura (HTS) sono in sviluppo, soprattutto perché sono più efficienti, e anche più leggeri. Il progetto Ecoswing, finanziato dall'UE, ha progettato il primo generatore HTS del mondo. Il generatore HTS è stato progettato per avere un diametro esterno limitato a soli $4 \mathrm{~m}$ e un peso di 68,75 tonnellate, un $24 \%$ più leggero da un PM generatore delle stesse caratteristiche, con un potenziale di 
raggiungere il $40 \%$ nei progetti futuri ${ }^{60}$.

- Utilizzare compositi ibridi (fibre di vetro e carbonio), rappresenta un'interessante alternativa ai rinforzi in fibra di carbonio pura ${ }^{61}$. Uno studio ha dimostrato che utilizzando $100 \%$ fibre di carbonio invece di fibre di vetro per la produzione di pale eoliche si potrebbe raggiungere un risparmio di peso di circa l' $80 \%$, ma con un aumento dei costi del 141\%. Però, un rinforzo ibrido di 70\% GF e 30\% CF potrebbe significare una riduzione di peso del 50\% con solo un aumento di costo del $90 \%{ }^{62}$.

\section{Conclusioni}

Nei prossimi anni il settore dell'energia eolica sarà sfidata poiché molte turbine eoliche saranno dismesse. Pertanto, è fondamentale stabilire le linee guida necessarie per affrontare questo problema nel modo più ecologico possibile. Le normative locali e l'attuale contesto energetico ed economico, influenzeranno la decisione finale di fine vita di un parco eolico.

I progetti di recupero potrebbero essere una possibile soluzione per una percentuale delle quantità di rifiuti in arrivo, ma non per il volume totale previsto. Le diversi applicazioni devono essere valutate da diverse prospettive poiché gli aspetti tecnici, spaziali e ambientali e le valutazioni di accettazione sociale sono vitali per l'applicazione finale. Inoltre, i progetti innovativi devono essere sviluppati con ulteriori studi strutturali. A tal fine, i produttori delle turbine eoliche dovrebbero fornire maggiori informazioni sui loro modelli, in quanto la loro confidenzialità ha portato a fare molte ipotesi sulle diverse applicazioni e questo genera incertezze sui progetti. Tuttavia, il basso costo dei progetti potrebbe essere un aspetto essenziale per la fattibilità delle applicazioni.

Le tecnologie di riciclo dei materiali compositi sono una possibile soluzione anche se ancora in fase di sviluppo, perché la loro efficacia nell'estrazione di fibre e matrici con buone proprietà meccaniche a prezzi competitivi è bassa. Il problema dei rifiuti compositi non è l'unico per l'industria 
eolica poiché i materiali FRP sono stati sempre utilizzati in altri settori, come aerospaziale, automobilistico, marino, delle costruzioni e degli sports. Pertanto, è possibile stabilire un'industria globale del riciclo dei rifiuti con processi di selezione e separazione dei materiali ben definiti per migliorare le prestazioni dei diversi metodi.

È necessario definire una politica globale per trattare questa problematica. Extended Producer Responsability è definito come "un approccio di politica ambientale in cui la responsabilità di un produttore per un prodotto è estesa alla fase post-consumo del ciclo di vita del prodotto" 63 . La responsabilità non solo dovrebbe ricadere nei produttori, ma anche i governi dovrebbero incentivare i produttori a tenere conto degli aspetti ambientali nella progettazione delle turbine.

La migliore strategia per le pale delle turbine eoliche è quella che combina designi ecologici e con materiali più resistenti, servizi di manutenzione regolari con una operazione sistematica. Tutto questo insieme porterà a raggiungere un'economia circolare ${ }^{64}$, con un impatto ambientale minore e, pertanto, potrebbe contribuire a raggiungere gli obiettivi ambientali stabiliti dalla Commissione Europea.

\section{References}

1.Scannapieco, D., Naddeo, V. \& Belgiorno, V. Sustainable power plants: A support tool for the analysis of alternatives. Land Use Policy 36, 478-484 (2014).

2.BARBIR, F., VEZIROGLU, T. \& PLASSJR, H. Environmental damage due to fossil fuels use. International Journal of Hydrogen Energy 15, 739-749 (1990).

3.Omer, A. M. Energy environment and sustainable development. Renewable and Sustainable Energy Reviews 12, 2265-2300 (2008).

4.Panwar, N. L., Kaushik, S. C. \& Kothari, S. Role of renewable energy sources in environmental 
protection: A review. Renewable and Sustainable Energy Reviews 15, 1513-1524 (2011).

5.Zarra, T., Galang, M. G., Ballesteros, F., Belgiorno, V. \& Naddeo, V. Environmental odour management by artificial neural network - A review. Environment International 133, 105189 (2019).

6.Zarra, T., Naddeo, V. \& Belgiorno, V. A novel tool for estimating the odour emissions of composting plants in air pollution management. Global Nest Journal 11, 477-486 (2009).

7.Naddeo, V., Zarra, T., Giuliani, S. \& Belgiorno, V. Odour impact assessment in industrial areas. CHEMICAL ENGINEERING 30, (2012).

8.Bassano, N. Sostenibilità ambientale degli impianti di produzione di energia. (2020) doi:10.22541/au.158100381.13374115.

9.Nesticò, A., Elia, C. \& Naddeo, V. Sustainability of urban regeneration projects: Novel selection model based on analytic network process and zero-one goal programming. Land Use Policy 99, $104831(2020)$.

10.Naddeo, V., Belgiorno, V., Zarra, T. \& Scannapieco, D. Dynamic and embedded evaluation procedure for strategic environmental assessment. Land Use Policy 31, 605-612 (2013).

11.Naddeo, V. \& Korshin, G. Water energy and waste: The great European deal for the environment. Science of The Total Environment 764, 142911 (2021).

12.Naddeo, V. Frontiers in Water-Energy-Nexus-Nature-Based Solutions Advanced Technologies and Best Practices for Environmental Sustainability. (Springer International Publishing, 2020). doi:10.1007/978-3-030-13068-8.

13.Hasan, S. W. Environmental Technologies for the Sustainable Development of the Water and Energy Sectors. (2020) doi:10.2166/9781789062328.

14.Murena, A. et al.. Water-Energy Nexus: Evaluation of the Environmental Impact on the Na- 
tional and International Scenarios. in Frontiers in Water-Energy-Nexus-Nature-Based Solutions Advanced Technologies and Best Practices for Environmental Sustainability 33-35 (Springer International Publishing, 2019). doi:10.1007/978-3-030-13068-8.

15.Cerullo, F. \& Sabatano, L. Energia eolica: impatti positivi e negativi su vegetazione, flora e fauna. (2021) doi:10.22541/au.161801138.81674455/v1.

16.Bianco, S., Cannavale, B. I. \& Vecchio, C. La Valutazione d'Incidenza Ambientale: tra conservazione degli habitat e gestione sostenibile dei rifiuti doi:10.22541/au.161437487.74855935/v1.

17.Carrara, S., ALVES DIAS, P., Plazzotta, B. \& Pavel, C. Raw materials demand for wind and solar PV technologies in the transition towards a decarbonised energy system. (2020).

18.Jensen, J. P. \& Skelton, K. Wind turbine blade recycling: Experiences challenges and possibilities in a circular economy. Renewable and Sustainable Energy Reviews 97, 165-176 (2018).

19.Serri, L., Lembo, E., Airoldi, D., Gelli, C. \& Beccarello, M. Wind energy plants repowering potential in Italy: technical-economic assessment. Renewable Energy 115, 382-390 (2018).

20.de Bona, J. C., Ferreira, J. C. E. \& Ordoñez Duran, J. F. Analysis of scenarios for repowering wind farms in Brazil. Renewable and Sustainable Energy Reviews 135, 110197 (2021).

21.Castro-Santos, L., Filgueira, A., Camacho, E. \& Piegiari, L. Costs and feasibility of repowering wind farms. Energy Sources, Part B: Economics, Planning, and Policy 11, 974-981 (2016).

22.Ziegler, L., Gonzalez, E., Rubert, T., Smolka, U. \& Melero, J. J. Lifetime extension of onshore wind turbines: A review covering Germany, Spain, Denmark, and the UK. Renewable and Sustainable Energy Reviews 82, 1261-1271 (2018).

23.Besseau, R., Sacchi, R., Blanc, I. \& Pérez-López, P. Past, present and future environmental footprint of the Danish wind turbine fleet with 
$\mathrm{LCA}_{W} I N D_{D} K$, anonlineinteractiveplat form.Renewable and Sustainable Energy Reviews108, 274-288(2019

24.Suhail, R., Chen, J.-F., Gentry, R. \& Bank, L. Analysis and Design of Pedestrian Bridge with Decommissioned FRP Windblades. (2019) doi:10.13140/RG.2.2.19230.36165.

25.Gentry, R. et al.. Structural Analysis of a Roof Extracted from a Wind Turbine Blade. Journal of Architectural Engineering 26, 04020040 (2020).

26.Joustra, J., Flipsen, B. \& Balkenende, R. Structural reuse of high end composite products: A design case study on wind turbine blades. Resources, Conservation and Recycling 167, 105393 (2021).

27.Yazdanbakhsh, A., Bank, L. C., Rieder, K.-A., Tian, Y. \& Chen, C. Concrete with discrete slender elements from mechanically recycled wind turbine blades. Resources, Conservation and Recycling 128, 11-21 (2018).

28.Estanqueiro, B., Silvestre, J., Brito, J. \& Pinheiro, M. Environmental life cycle assessment of coarse natural and recycled aggregates for concrete. European Journal of Environmental and Civil Engineering 1-21 (2018) doi:10.1080/19648189.2016.1197161.

29.Yazdanbakhsh, A., Bank, L., Baez, T. \& Wernick, I. Comparative LCA of concrete with natural and recycled coarse aggregate in the New York City area. The International Journal of Life Cycle Assessment 23, (2016).

30.Su, X. Electrochemical separations for metal recycling. Electrochemical Society Interface 29, $55-61(2020)$.

31.Panayotova, M. Metals Recycling. in (2017). doi:10.1002/0471238961.1415140618090308.a01.pub3.

32.Jowitt, S. M., Werner, T. T., Weng, Z. \& Mudd, G. M. Recycling of the rare earth elements. Current Opinion in Green and Sustainable Chemistry 13, 1-7 (2018).

33.Binnemans, K. et al.. Recycling of rare earths: a critical review. Journal of Cleaner Production 
51, 1-22 (2013).

34.Nagle, A. J., Delaney, E. L., Bank, L. C. \& Leahy, P. G. A Comparative Life Cycle Assessment between landfilling and Co-Processing of waste from decommissioned Irish wind turbine blades. Journal of Cleaner Production 277, 123321 (2020).

35.Pickering, S. J. Recycling technologies for thermoset composite materials-current status. Composites Part A: Applied Science and Manufacturing 37, 1206-1215 (2006).

36.Chen, J., Wang, J. \& Ni, A. Recycling and reuse of composite materials for wind turbine blades: An overview. Journal of Reinforced Plastics and Composites 38, 073168441983347 (2019).

37.Correia, J. R., Almeida, N. M. \& Figueira, J. R. Recycling of FRP composites: reusing fine GFRP waste in concrete mixtures. Journal of Cleaner Production 19, 1745-1753 (2011).

38.Zhang, J., Chevali, V. S., Wang, H. \& Wang, C.-H. Current status of carbon fibre and carbon fibre composites recycling. Composites Part B: Engineering 193, 108053 (2020).

39.Moraes, V., Jermolovicius, L., Tenório, J., Lebrão, S. \& Lebrão, G. Microwave-Assisted Recycling Process to Recover Fiber from Fiberglass Polyester Composites. Materials Research 22, (2019).

40.Oliveux, G., Dandy, L. O. \& Leeke, G. A. Current status of recycling of fibre reinforced polymers: Review of technologies, reuse and resulting properties. Progress in Materials Science 72, 61-99 (2015).

41.Cunliffe-Williams. Characterisation of products from the recycling of glass fibre reinforced polyester waste by pyrolysis. Fuel 82, 2223-2230 (2003).

42.Cunliffe, A., Jones, N. \& Williams, P. Pyrolysis of composite plastic waste. Environmental technology 24, 653-63 (2003).

43.Leißner, T., Hamann, D., Wuschke, L., Jäckel, H.-G. \& Peuker, U. A. High voltage fragmenta- 
tion of composites from secondary raw materials - Potential and limitations. Waste Management 74, (2018).

44.Mativenga, P. T., Shuaib, N. A., Howarth, J., Pestalozzi, F. \& Woidasky, J. High voltage fragmentation and mechanical recycling of glass fibre thermoset composite. CIRP Annals 65, 45-48 (2016).

45.Lee, S.-H. et al.. Circulating flow reactor for recycling of carbon fiber from carbon fiber reinforced epoxy composite. Korean Journal of Chemical Engineering - KOREAN J CHEM ENG 28, 449-454 (2011).

46.Yuyan, L., Guohua, S. \& Linghui, M. Recycling of carbon fibre reinforced composites using water in subcritical conditions. Materials Science and Engineering: A 520, 179-183 (2009).

47.Pimenta, S. \& Pinho, S. T. Recycling carbon fibre reinforced polymers for structural applications: Technology review and market outlook. Waste Management 31, 378-392 (2011).

48.Cousins, D. S., Suzuki, Y., Murray, R. E., Samaniuk, J. R. \& Stebner, A. P. Recycling glass fiber thermoplastic composites from wind turbine blades. Journal of Cleaner Production 209, $1252-1263$ (2019).

49.Sokoli, H., Simonsen, M. \& Sogaard, E. Investigation of degradation products produced by recycling the solvent during chemical degradation of fiber-reinforced composites. Journal of Reinforced Plastics and Composites 36, 073168441770706 (2017).

50.Piñero-Hernanz, R. et al. . Chemical recycling of carbon fibre composites using alcohols under subcritical and supercritical conditions. The Journal of Supercritical Fluids 46, 83-92 (2008).

51.Pickering, S. J., Kelly, R. M., Kennerley, J. R., Rudd, C. D. \& Fenwick, N. J. A fluidised-bed process for the recovery of glass fibres from scrap thermoset composites. Composites Science and Technology 60, 509-523 (2000). 
52.Kennerley, J. R., Kelly, R. M., Fenwick, N. J., Pickering, S. J. \& Rudd, C. D. The characterisation and reuse of glass fibres recycled from scrap composites by the action of a fluidised bed process. Composites Part A: Applied Science and Manufacturing 29, 839-845 (1998).

53.Pender, K. \& Yang, L. Regenerating performance of glass fibre recycled from wind turbine blade. Composites Part B: Engineering 198, 108230 (2020).

54.Yip, H. L. H., Pickering, S. J. \& Rudd, C. D. Characterisation of carbon fibres recycled from scrap composites using fluidised bed process. Plastics Rubber and Composites 31, 278-282 (2002).

55.Åkesson, D., Foltynowicz, Z., Christéen, J. \& Skrifvars, M. Microwave pyrolysis as a method of recycling glass fibre from used blades of wind turbines. Journal of Reinforced Plastics and Composites 31, 1136-1142 (2012).

56.Obunai, K., Fukuta, T. \& Ozaki, K. Carbon fiber extraction from waste CFRP by microwave irradiation. Composites Part A: Applied Science and Manufacturing 78, 160-165 (2015).

57.Jiang, L. et al.. Recycling carbon fiber composites using microwave irradiation: Reinforcement study of the recycled fiber in new composites. Journal of Applied Polymer Science 132, n/a-n/a (2015).

58.Li, Y. \& Lu, J. Lightweight structure design for wind energy by integrating nanostructured materials. Materials \& Design 57, 689-696 (2014).

59.Goudarzi, M., Stonis, M. \& Behrens, B.-A. Development of Lightweight Designs for the Production of Wind Turbine Towers. in Enabling Manufacturing Competitiveness and Economic Sustainability 443-448 (Springer, 2014).

60.Song, X. et al.. Designing and Basic Experimental Validation of the World's First MW-Class Direct-Drive Superconducting Wind Turbine Generator. IEEE Transactions on Energy Conversion 
34, 2218-2225 (2019).

61.Mishnaevsky, L. et al.. Materials for Wind Turbine Blades: An Overview. Materials 10, 1285 (2017).

62.Ong, C.-H. \& Tsai, S. W. The use of carbon fibers in wind turbine blade design: a SERI-8 blade example. (2000).

63.OECD. Extended Producer Responsibility. (OECD, 2001). doi:10.1787/9789264189867-en.

64.Naddeo, V. \& Taherzadeh, M. J. Biomass valorization and bioenergy in the blue circular economy. Biomass and Bioenergy 149, 106069 (2021). 


\section{Figure Captions}

Figure 1. Parco eolico (fonte: unplash.com)

Figure 2. Materiali presenti in una turbina eolica. (fonte: WindEurope-Decommissioning of Onshore Wind Turbines).

Figure 3. Gerarchia dei tratamenti dei rifiuti. (fonte: Suschem-Polymer Composites Circularity).

Figure 4. Progetti di recupero. 
Figures

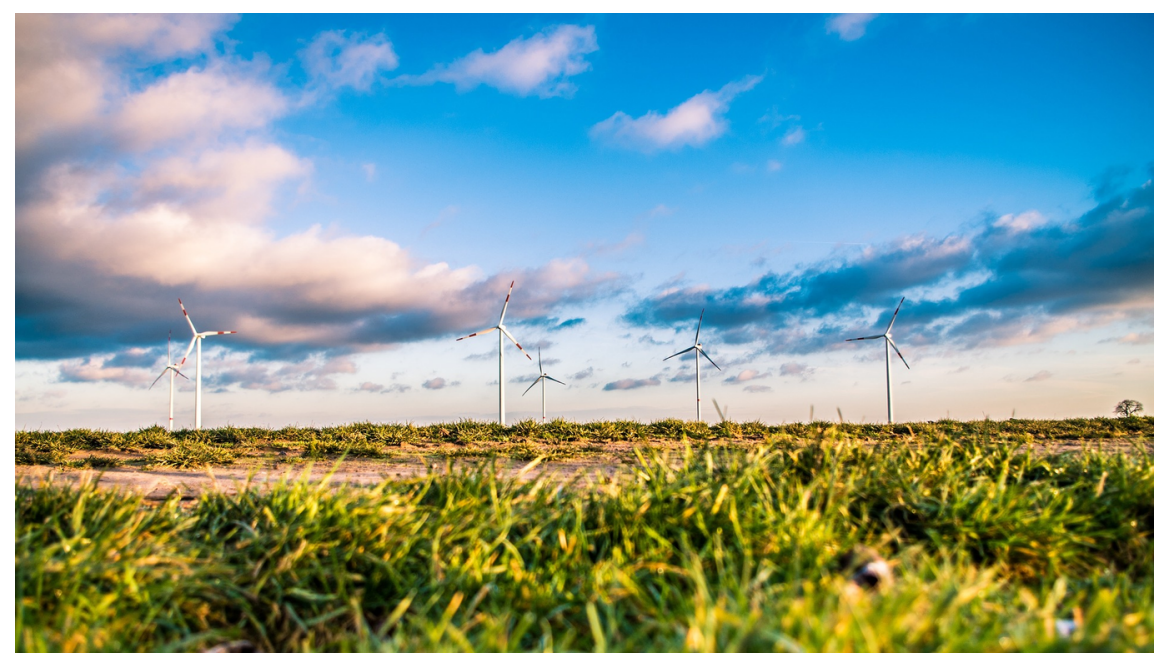

Figure 1: Parco eolico (fonte: unplash.com) 


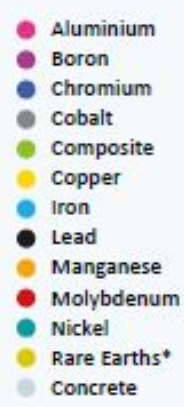

- Dysprosium, neodymium, praseodymium, terbium

Sources: European commission Materials Information System (MIS) Wind Energy, April 2016 and European commission Raw Materials Scoreboard 2016.

The source does not specify the wind turbine on which the above information is based.

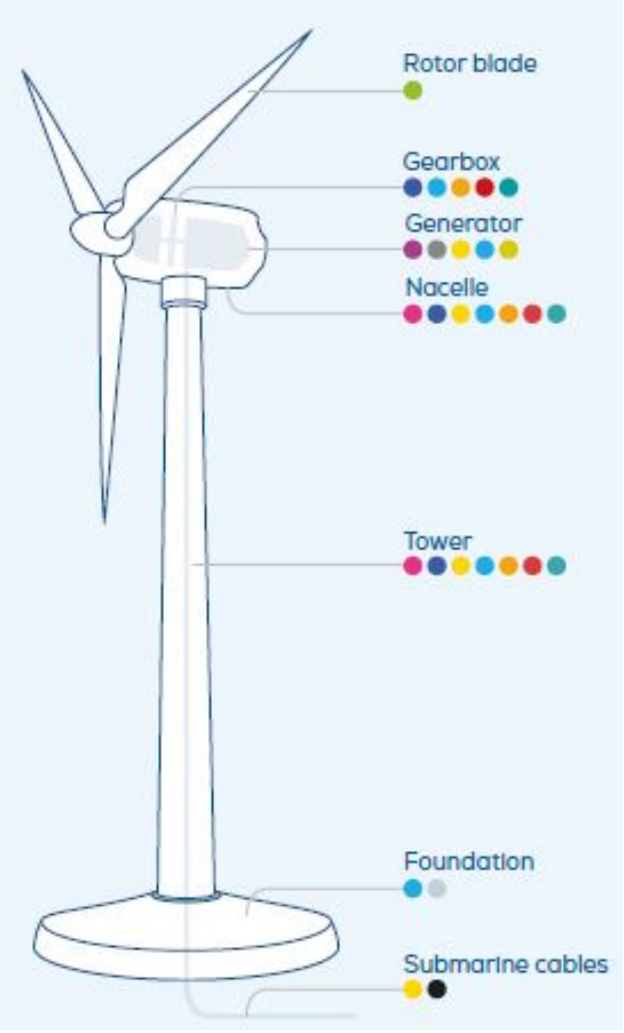

Figure 2: Materiali presenti in una turbina eolica. (fonte: WindEurope-Decommissioning of Onshore Wind Turbines).

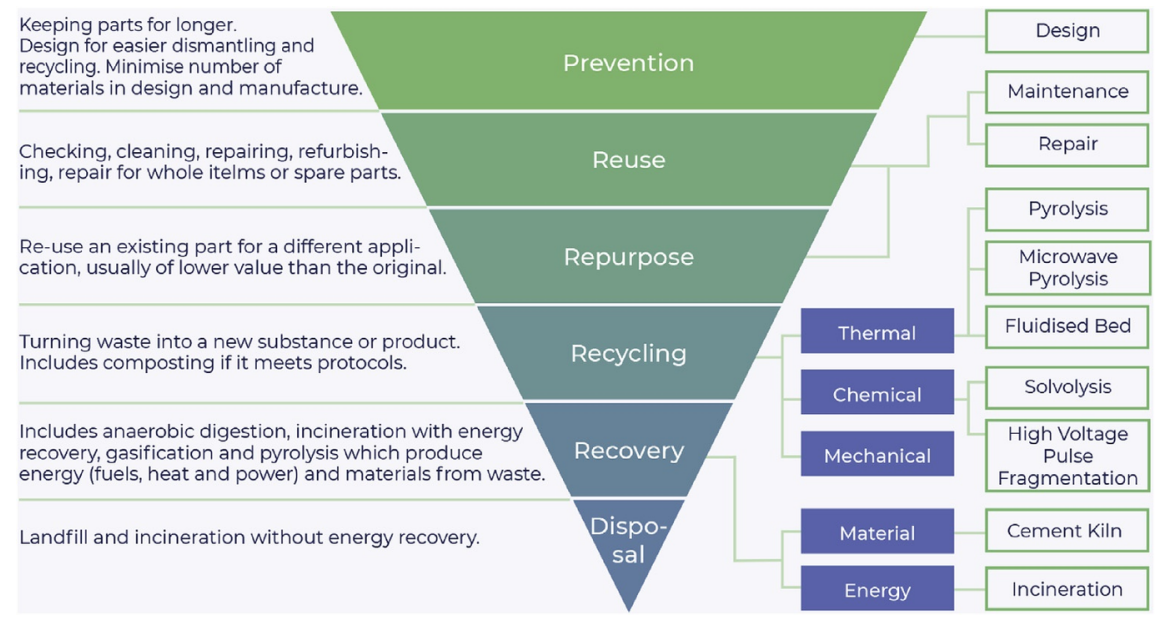

Figure 3: Gerarchia dei tratamenti dei rifiuti. (fonte: Suschem-Polymer Composites Circularity). 


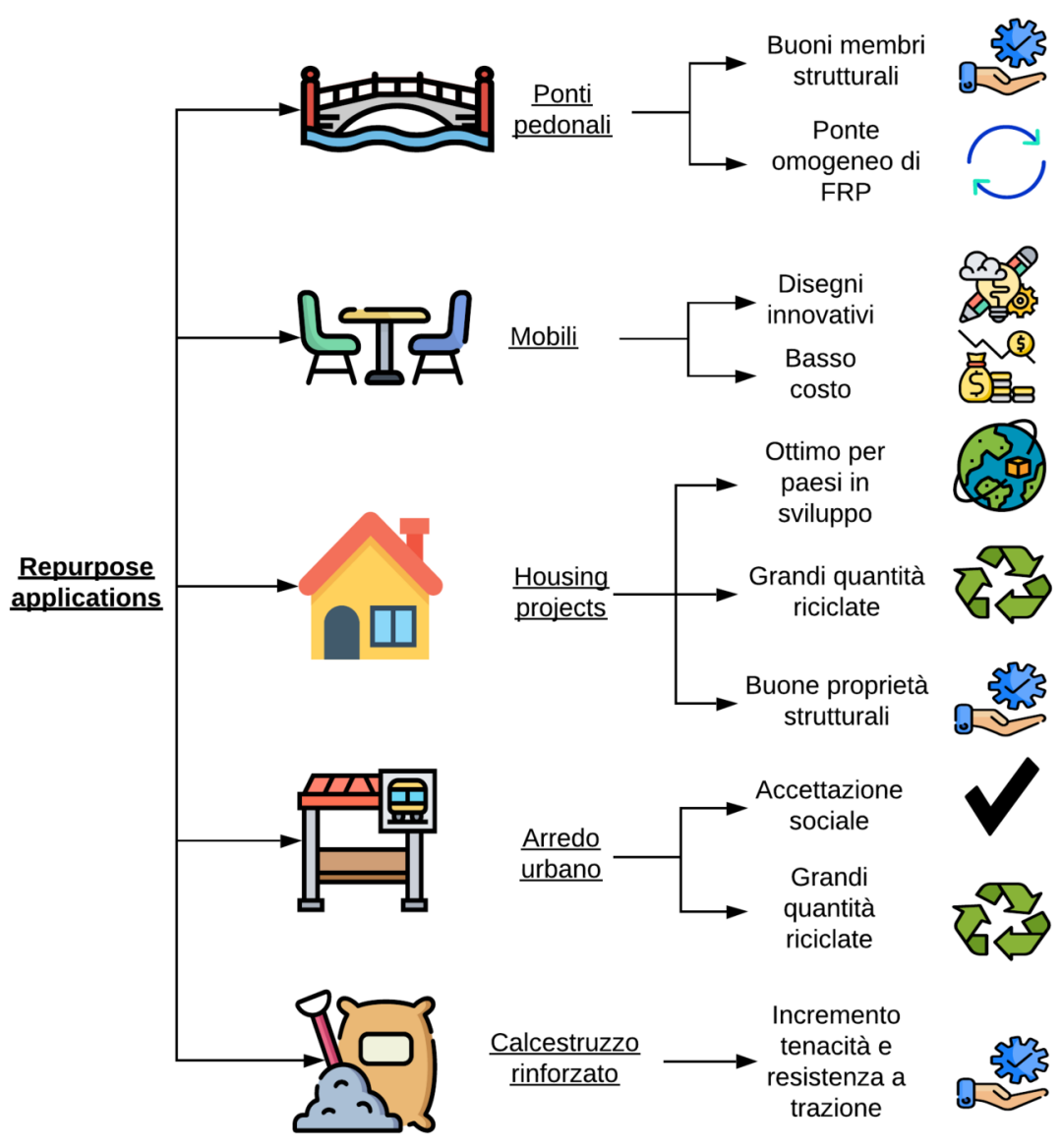

Figure 4: Progetti di recupero. 